\title{
AS \& T- T: \\ Estudo do uso de agente secante na obtenção de vidros via método sol-gel
}

\author{
Gilberto Jorge Peçanha ${ }^{1}$, Florêncio Gomes de Ramos Filho ${ }^{1}$, Shirleny Fontes Santos ${ }^{1 *}$
}

\section{AUTHOR AFILIATIONS}

1 - Centro Universitário Estadual da Zona Oeste (UEZO), 23070-200,

Rio de Janeiro (RJ), Brasil

\section{CONTACT}

lenyfontes@yahoo.com.br

shirleny@uezo.rj,gov.br

\section{ABSTRACT}

Vitreous ceramic materials obtained in the form of membranes can be used as filter media, among other applications. The applicability of these materials depends, among other things, on the size and quantity of the pores and the mechanical resistance. The sol-gel method has been studied as a potential alternative for obtaining materials with uniform morphology without breaking and cracking. In this context, silica-based glass membranes $\left(\mathrm{SiO}_{2}\right)$ were prepared by the sol-gel method, using Tetraethylstilicate (TEOS) as precursor and N, Ndimethylformamide (DMF) as a drying control agent. The samples were characterized by X-ray diffraction, which evidenced the presence of amorphous structure. Thermal analyzes (TG / DSC) indicated that the presence of DMF slightly decreased the temperatures of the thermal events of the membrane. The porosity measurement by the Archimedes method indicated about $65 \%$ of porosity to the monoliths obtained with use of DMF.

Keywords: Sol-gel, vitreous membranes, DMF.

\section{RESUMO}

Materiais cerâmicos vítreos obtidos na forma de membranas podem ser utilizados como meios filtrantes, entre outras aplicações. A aplicabilidade desses materiais depende, entre outras coisas, do tamanho e quantidade dos poros e da resistência mecânica. O método sol-gel tem sido estudado como alternativa promissora para a obtenção de materiais com morfologia uniforme sem a presença de trincas e rachaduras. Sob esta perspectiva, foram preparadas membranas cerâmicas vítreas com base em sílica $\left(\mathrm{SiO}_{2}\right)$ pelo método sol-gel, utilizando o precursor Tetraetilortossilicato (TEOS) e com uso de N,N-dimetilformamida (DMF) como agente de controle da secagem. As amostras obtidas foram caracterizadas por difração de raios-X, que evidenciou a presença de estrutura amorfa. As análises térmicas (TG/DSC) indicaram que a presença do DMF diminui ligeiramente as temperaturas nos quais os eventos térmicos da membrana ocorrem. A medida de porosidade pelo Método de Arquimedes indicou que os monolitos obtidos com uso de DMF apresentaram em seu estado pré-calcinado cerca de $65 \%$ de porosidade. 
Palavras-chaves: Sol-gel, membrana vítrea, DMF.

\section{INTRODUÇÃO}

Membranas são usualmente definidas como filtros ou barreiras que separam duas fases, restringindo, total ou parcialmente, o transporte de uma ou várias espécies químicas presentes nas fases. As membranas podem ser poliméricas (orgânicas), inorgânicas (cerâmicas) ou híbridas (orgânica/inorgânica). As membranas de natureza orgânica são mais baratas e podem ser facilmente aplicadas. As inorgânicas, por outro lado, devido a sua elevada resistência mecânica e estabilidade térmica e química, vêm ganhando atenção dos pesquisadores e das indústrias (TSURU et al, 2008; SILVA et al, 2014).
Dentre as membranas inorgânicas destacam-se aquelas com base em alumina $\left(\mathrm{Al}_{2} \mathrm{O}_{3}\right)$, óxido de silício $\left(\mathrm{SiO}_{2}\right)$, zircônia $\left(\mathrm{ZrO}_{2}\right)$ e titânia $\left(\mathrm{TiO}_{2}\right)$. As mesmas podem ser amorfas, usualmente preparadas com base em $\mathrm{SiO}_{2}$, ou cristalinas, tais como as de alumina, zircônia e titânia ou composições mistas envolvendo mais de um material cerâmico, como estruturas de $\mathrm{SiO}_{2}-\mathrm{Al}_{2} \mathrm{O}_{3}$ e $\mathrm{TiO}_{2} / \mathrm{ZnAl}_{2} \mathrm{O}_{4}$ (CHAVES et al, 2013).

O campo de aplicação para todos os tipos de membranas é vasto, porém sua aplicação mais óbvia inclui justamente os processos de filtração (ultra, micro e nanofiltração), com aplicação prática no tratamento de água e alimentos, recuperação de $\mathrm{H}_{2}$, $\mathrm{He}$ e $\mathrm{CO}_{2}$, processamento de 
misturas orgânicas, entre outros (TSURU et al, 2008; ALEM et al, 2009; SILVA et al, 2014).

No que concerne aos métodos de obtenção particularmente de membranas inorgânicas podem ser citar: extrusão, spin coating, irradiação, sinterização, entre outros. Entretanto novos métodos que possibilitem obtenção de materiais de forma mais rápida, com características melhoradas e menor custo vêm sendo constantemente investigados (GOMES et al, 2014).

A possibilidade de controlar a geometria e rede de poros, aliada ao alto desempenho estrutural das cerâmicas obtidas pelo método solgel, tornam este método uma possibilidade atrativa para a obtenção de membranas tanto inorgânicas, quanto híbridas (VIART et al, 1997).

O processo sol-gel envolve a obtenção de uma matriz inorgânica através da formação de um sol e sua transformação em um gel úmido, que após a retirada do liquido forma o gel seco e poroso que pode ser sinterizado em sólido denso e amorfo. (BETANCOURT-PARRA et al, 2019). Entretanto, uma das grandes dificuldades no preparo de monolitos pelo método sol-gel diz respeito ao surgimento de trincas que podem levar a quebra do material. Aditivos químicos para controle da secagem podem ser adicionados, a fim de reduzir o tempo de processamento e evitar o aparecimento de trincas durante a etapa de secagem (SANTOS et al, 2007).

Neste contexto, o objetivo deste trabalho consistiu na síntese e caracterização de membranas inorgânicas vítreas $\left(\mathrm{SiO}_{2}\right)$ pelo método sol-gel com estudo da influência da adição do agente secante N,N-dimetil formamida (DMF).

\section{METODOLOGIA}

\section{Síntese das membranas de $\mathrm{SIO}_{2}$}

Para síntese das membranas de $\mathrm{SiO}_{2}$ calculou-se a relação estequiométrica entre os reagentes precursores adequada para obtenção de um gel denso: TEOS: $\mathrm{H}_{2} \mathrm{O}: \mathrm{C}_{2} \mathrm{H}_{5} \mathrm{OH}, 1: 10: 6$. Com base nessa relação, adicionaram-se os reagentes TEOS (tetraetilortosilicato, Sigma-Aldrich, 98\%), água destilada e etanol (VETEC, 99\%) em um béquer, o $\mathrm{pH}$ do sistema foi ajustado para 1,0 por meio da adição de solução a $1 \mathrm{M}$ de ácido 
clorídrico. O sistema foi deixado para envelhecer a temperatura ambiente por 4 semanas.

Foi preparado também um experimento $\begin{array}{lll}\text { com } & \text { adição } & \text { DMF }\end{array}$ dimetilformamida,Sigma-Aldrich, 99\%) que é um agente de controle da secagem. Para este sistema a relação estequiométrica foi de: TEOS: $\mathrm{H}_{2} \mathrm{O}: \mathrm{C}_{2} \mathrm{H}_{5} \mathrm{OH}: D M F, \quad 1: 10: 6: 1$. Esta mistura também foi mantida a temperatura ambiente para envelhecimento por 4 semanas.

\section{TÉCNICAS DE CARACTERIZAÇÃO}

A estrutura cristalográfica das membranas foi determinada por difratômetria de raios-X, com radiação Co $\mathrm{K} \alpha(35 \mathrm{kV} / 40 \mathrm{~mA}) ; 2 \theta$ na faixa de $5^{\circ}$ a $80^{\circ}$.

As curvas de TGA/DSC das membranas sintetizadas foram obtidas por meio de um equipamento SDT Q600 V20.9 Build 20 da TA Instruments. Utilizaram-se amostras na forma de pó maceradas com a ajuda do almofariz, atmosfera de nitrogênio e cadinho de platina. A taxa de aquecimento foi de $10^{\circ} \mathrm{C} / \mathrm{min}$ e a faixa de temperatura do ensaio foi de cerca de 30 à $1200^{\circ} \mathrm{C}$.
Para os ensaios de porosimetria de imersão (método de Arquimedes), utilizou-se a equação 1:. $\left[\mathrm{M}_{\mathrm{s}} /\left(\mathrm{M}_{\mathrm{u}}-\mathrm{M}_{\mathrm{ap}}\right)\right] \times \mathrm{\rho H}_{2} \mathrm{O}$. Onde $\mathrm{M}_{\mathrm{s}}$ é a massa seca da membrana, $\mathrm{M}_{\mathrm{u}}$ corresponde a massa úmida e $\mathrm{M}_{\mathrm{ap}}$ é a massa aparente. Foi verificado que a densidade da água destilada estava em torno de $0,9971 \mathrm{~g} \cdot \mathrm{cm}^{-3}$ e a temperatura a $25^{\circ} \mathrm{C}$.

\section{RESULTADOS E DISCUSSÕES}

\section{DIFRAÇÃO DE RAIOS-X}

Foram realizadas as difrações de raios-X das membranas de sílica com e sem DMF, conforme mostrado na Figura 1 (a) e (b). Pôde-se verificar a ausência de picos relacionados à formação de estrutura cristalina, verifica-se apenas a presença de um halo a cerca de $30^{\circ}$ típico da formação de sílica vítrea (não-cristalina), (SANTOS et al, 2007), verificou-se também que o DMF não alterou a estrutura amorfa obtida, pois as amostras com e sem DMF apresentaram perfis bastantes similares. 

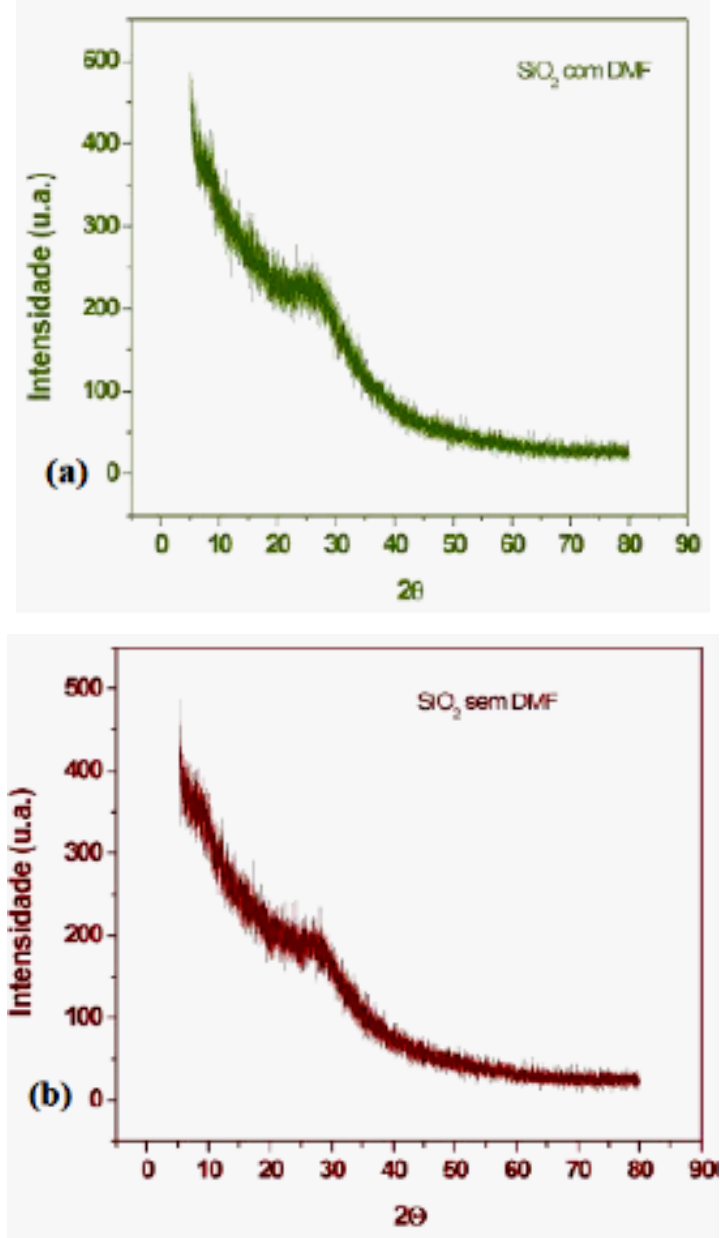

Figura 1 - DRX das membranas (a) com DMF (b) sem DMF.

\section{Análises Térmicas}

A Figura 2 ilustra os termogramas de TGA obtidos para as membranas sintetizadas com e sem DMF. Os perfis térmicos para duas amostras revelam que ambas as membranas apresentaram uma curva de perda de massa com dois estágios de decomposição (LENZA et al, 2002).

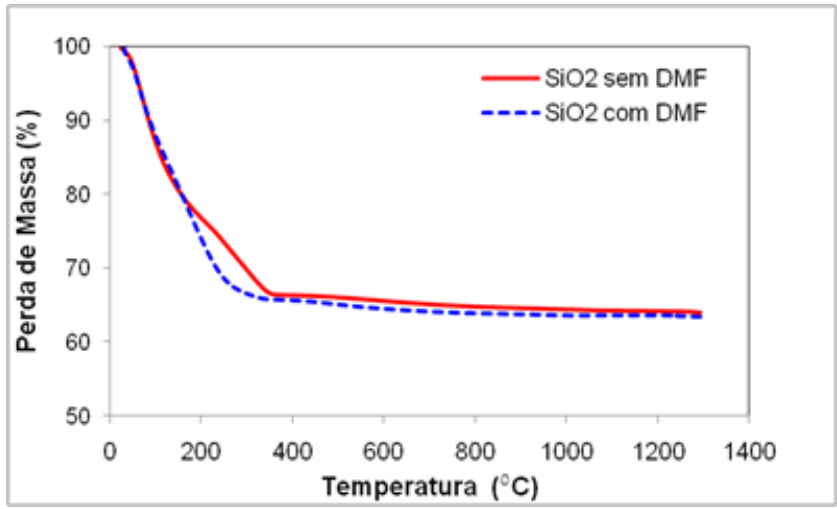

Figura 2 - Curva de Termogravimetria (TG) das membranas de sílica com e sem DMF.

A primeira até cerca de $200^{\circ} \mathrm{C}$, que está relacionada com a perda de água fisicamente adsorvida. A segunda, até cerca de $400^{\circ} \mathrm{C}$ é relacionada com a perda de água quimicamente adsorvida, grupos silanol superficiais, e perda de materiais de partida não reagidos (LENZA et al, 2002). Para este segundo estágio observa-se que a amostra com DMF exibiu perda de massa em temperaturas inferiores à da amostra sem DMF, o que sugere que este agente secante atua interagindo com os subprodutos de reação tornando-os mais voláteis.

As curvas de DSC, Figura 3, exibem um pico endotérmico a cerca de $430{ }^{\circ} \mathrm{C}$, correspondente ao fenômeno de saída de água. $\mathrm{O}$ perfil da amostra com DMF exibe ainda um discreto pico exotérmico próximo a $1000^{\circ} \mathrm{C}$ e 
ainda um pico endotérmico a cerca de $1300{ }^{\circ} \mathrm{C}$, possivelmente relacionado a um processo de cristalização seguido de fusão (LENZA et al, 2002; SANTOS et al, 2007).

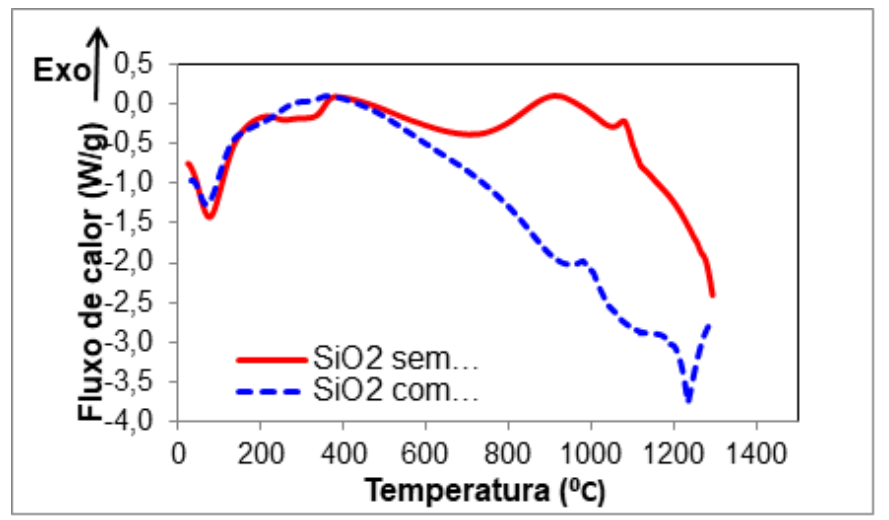

Figura 3 - Curva de calorimetria diferencial (DSC) das amostras de membranas sintetizadas sem DMF e com DMF.

Os mesmos eventos podem ser observados no perfil da amostra sem DMF, no entanto em temperaturas superiores, inclusive o pico de fusão parece ter se iniciado e sido interrompido antes da finalização do processo. Este resultado indica que o DMF pode ter contribuído para alterar a cinética de cristalização e fusão desta membrana (LENZA et al, 2002; SANTOS et al, 2007).

\section{TESTE DE POROSIDADE}

A imagem das membranas com e sem DMF podem ser observadas na Figura 4 (a) e (b), em que se observa que após a adição do agente a quantidade de rachaduras foi significativamente reduzida.

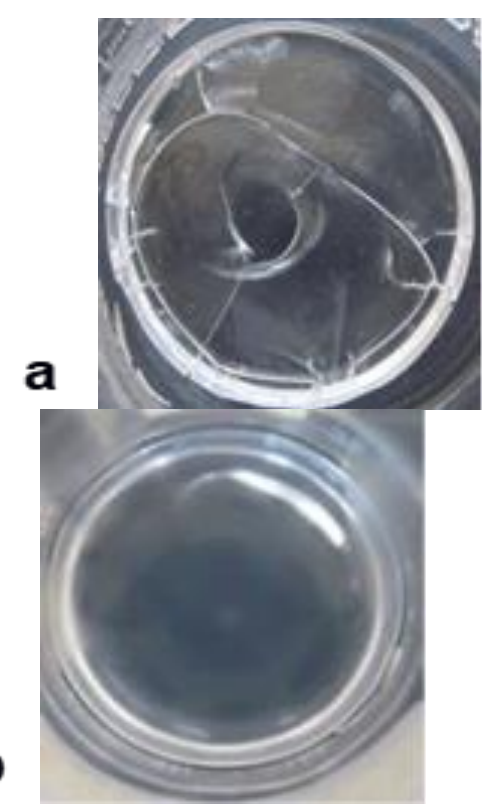

Figura 4 - Membranas de $\mathrm{SiO}_{2}$ (a) sem DMF e (b) com DMF.

Realizaram-se medidas da espessura das membranas no início da etapa de gelação e após quatro semanas de envelhecimento quando ocorreu a formação do monolito seco (nãocalcinado) em que verificou-se que para ambas as amostras ocorreu uma diminuição (encolhimento) de cerca de $50 \%$ da espessura. 
Tabela 1 - Percentual de encolhimento das membranas por medidas

da

espessura.

\begin{tabular}{|c|c|}
\hline \multicolumn{2}{|c|}{ Encolhimento sem DMF } \\
\hline Espessuras (mm) & Percentual de redução (\%) \\
& 53,3 \\
\hline Inicial: 42,51 & 530 \\
Final: 22,69 & Percentual de redução (\%) \\
Espessuras (mm) & 55,7 \\
Inicial: 42,51 & \\
Final: 23,68 & \\
\hline
\end{tabular}

Observa-se pelos dados da tabela que o DMF não alterou significativamente a porcentagem de escolhimento das membranas, apenas atuou controlando a secagem e gerando um material mais homogêneo e menos quebradiço.

A porcentagem de porosidade obtida pelo método de Arquimedes, análise revelou que a membrana de sílica com DMF apresenta cerca de $65 \%$ de porosidade, não foi possível realizar a análise da amostra sem DMF pois a mesma sofreu quebra significativa durante a tentativa de medida.

Além disso, avaliou-se a capacidade da membrana com DMF de absorver um outro material testando a colocação de um óleo industrial em sua estrutura e assim verificar a potencialidade deste material ser utilizado em sistemas filtrantes. As membranas foram submersas no óleo, Figura 5(a) e (b), que possui uma coloração amarelada e permaneceram submersas por 5 dias (120 horas).

a
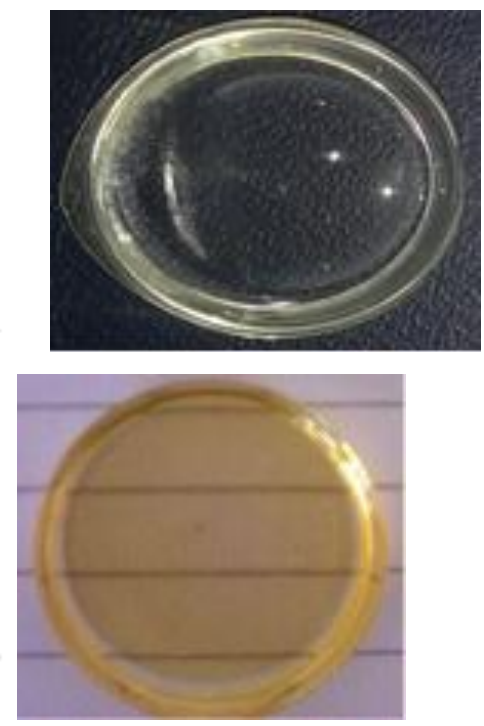

Figura 5. Sílica com DMF antes do ensaio (a) e após 5 dias de imersão em óleo (b).

Após o período de submersão por 120 horas foi possível verificar a mudança de coloração não somente na superfície, mas em toda membrana, evidenciando que houve interação entre os substratos do óleo com as membranas. Este 
resultado indica que a membrana apresenta uma porosidade aberta e com poros interconectados (REIMBRECHT et al, 2003) e sugere a potencialidade da membrana ser utilizada como meio filtrante.

A comparação do comportamento em óleo das membranas com e sem DMF reforça os dados já obtidos que indicam que o DMF não provoca alterações significativas nas membranas.

\section{CONCLUSÕES}

Membranas com base em sílica e com uso do N,N-dimetilformamida (DMF) como agente de controle da secagem foram preparadas pela metodologia sol-gel. O DMF se mostrou eficaz no controle da secagem e obtenção de material sem quebras.

A caracterização inicial das membranas (não-calcinadas) quanto a porosidade relevou material com cerca de $65 \%$ de porosidade do tipo aberta, com interconexão entre poros e entre estes e a superfície.

O estudo do comportamento térmicos dos materiais indicou a ação do DMF interagindo com os subprodutos da reação sol-gel e tornando-os mais voláteis para membranas puras.

O estudo realizado focou apenas na obtenção e caracterização das membranas, porém verificou-se que após maiores investigações este material poderá apresentar grandes potencialidades para chegar a ser utilizado em aplicações tecnológicas como em membranas para sistemas filtrantes.

\section{REFERÊNCIAS}

ALEM, A., SARPOOLAKY, H., KESHMIRI, M., 2009, Titania ultrafiltration membrane: Preparation, characterization, and photocatalytic activity, Journal of the European Ceramic Society 29, pp. 629-635.

CHAVES, A. C., LIRA, H. L, NEVES, G. A. et al, 2013, Obtenção e caracterização de membranas cerâmicas tubulares a partir de massas incorporadas com argila, caulim e quartzo, Cerâmica 59, n.349, pp. 192-197.

GOMES, A. C. O., MACHADO, I. M. M., FILHO, A. C. R., et al, 2014, Membranas híbridas com potencial uso em células a combustível - 
parte 2: nanocompósitos de poli(carbonato) sulfonado. Polímeros 24, n. 3, pp. 402-410.

LENZA, R. F. S.; VASCONCELOS, W. L., 2002, Síntese de membranas cerâmicas via método sol-gel utilizando TEOS e N, Ndimetilformamida. Química Nova 25, n. 6/A, pp. 893-896.

REIMBRECHT, E. G, BAZZO, E., ALMEIDA, L. H. S., et al, 2003, Manufacturing of metallic porous structures to be used in capillary pumping systems, Materials Research 6, n.4, pp. 481-486.

SANTOS, S. F., SANTOS, M. L., ALMEIDA, L. E. et al, 2007, Fluorescent Tetraruthenated Porphyrins Embedded In Monolithic $\mathrm{SiO}_{2}$ gels by the Sol-Gel Process, Journal of Colloid and Interface Science 305, pp. 264-269.

SILVA, M. C.; LIRA, H. L.; FREITAS, N. L., 2014, Membrana cerâmica assimétrica à base de argila para aplicação em processos de microfiltração: influência do tempo de deposição, Cerâmica 60, n. 355, pp.436-442.

TSURU, T., 2008, Nano/subnano-tuning of porous ceramic membranes for molecular separation, Journal of Sol-Gel Science and Technology 46, pp.349-361.

TSURU, T., NARITA, M., SHINAGAWA, R. et al, 2008, Nanoporous Titania Membranes for Permeation and Filtration of Organic Solutions, Desalination 233, n. 1-3, pp.1-9.

VIART, N., NIZNANSKY, D., REHSPRINGER, J. L., 1997, Magnetic Study of $\mathrm{SiO} 2 / \gamma-\mathrm{Fe}_{2} \mathrm{O}_{3}$ Nanocomposites prepared by the Sol-Gel method. Le Journal de Physique IV, v. 7, n. C1, pp. C1555-C1-556. 
Peçanha et al. 2020 